\title{
Bronquio cardíaco accesorio y otras variantes de la anatomía tráqueo-bronquial
}

\author{
TAMARA PALAVECINO B.* y RODRIGO PALMA C.**
}

\section{Accessory cardiac bronchus and others variations of the tracheobronchial anatomy}

Among the variations of bronchial anatomy, we can find dysplastic bronchial branches, an absence of bronchial branches, or supernumerary branches. Although many of them are asymptomatic, it is essential to know them because they may be associated with hemoptysis, recurrent infections, empyema, or complications secondary to various procedures (e.g. endotracheal intubation, brochoscopy, bronchoalveolar lavage, biopsy, endobronchial treatments, stent installation, brachytherapy and lung surgeries among others). In this study we used computed tomography to describe some of the most prevalent bronchial variations, including accessory cardiac bronchus, tracheal bronchus, and bridge bronchus among others.

Key words: Bronchi, bronchial disease, computed tomography.

\section{Resumen}

Entre las variantes de la anatomía bronquial nos podemos encontrar con ramas bronquiales displásicas, ramas ausentes o ramas supernumerarias. Aunque gran parte son asintomáticas, es esencial reconocerlas porque se pueden asociar a hemoptisis, infecciones recurrentes, empiema o a complicaciones secundarias a procedimientos (por ejemplo intubaciones endotraqueales, fibrobroncoscopías, lavado broncoalveolar, biopsias, tratamientos endobronquiales, instalación de stent, braquiterapia y cirugías pulmonares entre otros). En este artículo describimos las variantes bronquiales más prevalentes en tomografía computada, incluyendo al bronquio cardíaco accesorio, bronquio traqueal y bronquio puente, entre otras.

Palabras clave: Bronquios, enfermedades bronquiales, tomografía computada.

\section{Introducción}

Entre las variantes de la anatomía traqueobronquial podemos encontrar, por una parte, ramas bronquiales displásicas (lo más frecuente), que corresponden a un bronquio con origen anómalo, en ausencia del bronquio normal para ese segmento pulmonar y, por otro lado, con ramas supernumerarias, en las que el bronquio normal también está presente. Un bronquio supernumerario puede terminar en un fondo de saco ciego (llamados divertículos bronquiales congénitos) o puede ventilar parénquima, el que se considera tejido pulmonar adicional. También es posible la ausencia de alguna rama bronquial. Es relevante el conocimiento y la descripción adecuada de estas variantes y de las anomalías bronquiales previo a la realización de fibrobroncoscopías, lavado broncoalveolar, biopsias, tratamientos endobronquiales, instalación de stent, braquiterapia, resecciones pulmonares, trasplantes pulmonares, instalación de tubos endotraqueales entre otras ${ }^{1}$. Estas variantes tráqueo-bronquiales pueden existir de forma aislada o se pueden asociar a otras anomalías de la ramificación de la vía aérea, a enfermedades cardíacas congénitas, alteraciones costo-vertebrales, malformaciones del tubo digestivo, anomalías pulmonares o a síndromes genéticos ${ }^{2,3}$.

\footnotetext{
* Médico Radiólogo. Hospital Clínico Universidad de Chile. Santiago, Chile.

** Médico Residente de Radiología. Hospital Clínico Universidad de Chile. Santiago, Chile.
} 
Aunque las variaciones de la anatomía subsegmentaria son frecuentes, no suelen ser clínicamente significativas, pero pueden, por ejemplo, dificultar la realización de una broncoscopía o de un procedimiento quirúrgico al confundirse con algún reparo anatómico.

\section{Bronquio cardíaco accesorio}

El bronquio cardíaco accesorio es una variante poco frecuente que se presenta en un $0,08 \%$ de los pacientes que se realizan tomografías computadas de tórax ${ }^{1}$. Consiste en un bronquio supernumerario que nace de la pared medial del bronquio principal derecho o del bronquio intermedio, opuesto al origen del bronquio para el lóbulo superior derecho y proximal al bronquio del segmento apical del lóbulo inferior derecho (Figura 1), tiene una longitud variable y puede terminar en un fondo de saco ciego, sin tejido pulmonar asociado (Figura 2) o puede suplir un pequeño lóbulo ventilado de tejido pulmonar adicional, que puede estar separado del resto del parénquima por una cisura accesoria (Figuras 3, 4 y 5$)^{4,5}$. Se ha descrito su presentación simultánea con un bronquio traqueal o con un cartílago prominente a manera de espolón en la cara anterior de la carina ${ }^{6,7}$.

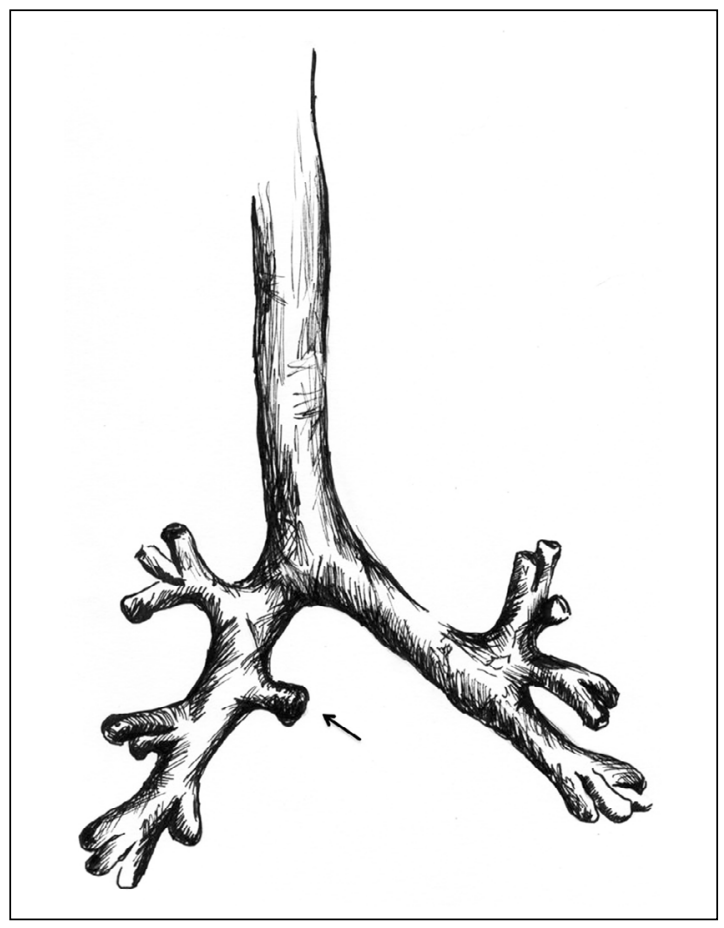

Figura 1. Esquema: Bronquio cardiaco accesorio (flecha).
En la mayoría de los casos son asintomáticos, pero se pueden asociar a hemoptisis, infecciones recurrentes o empiema ${ }^{5,8}$.

\section{Bronquio traqueal}

Los bronquios traqueales son variaciones comunes que corresponden a un bronquio superior derecho originado generalmente de la pared lateral derecha de la tráquea a menos de $2 \mathrm{~cm}$ sobre la carina, aireando el segmento apical del lóbulo superior (lo más frecuente) o el lóbulo superior completo ${ }^{1,9-11}$ (Figura 6 y 7). El bronquio traqueal puede ser displásico (más frecuente) o supernumerario $^{1,12}$, este último puede terminar en un fondo de saco ciego llamándose en este caso divertículo traqueal, o aireando un lóbulo hipoplásico $^{1,5,12}$. Se puede asociar a una disminución de calibre de la tráquea distal y generalmente no causa problemas, pero se puede asociar a infecciones (Figuras 8A y 8B), bronquiectasias, bronquitis crónica, enfisema focal, abscesos, quistes congénitos o atelectasias, atrapamiento aéreo y neumotórax secundario a intubación traqueal ${ }^{1,9,10,13,14}$, también puede acompañar a malformaciones cardiovasculares, síndromes genéticos y otras alteraciones de la segmentación pulmonar. Ruchonnet-Metrailler y cols describieron en su publicación que un $38,5 \%$ de los pacientes pediátricos evaluados en su serie con esta variante no tendrían malformaciones asociadas $^{3}$.

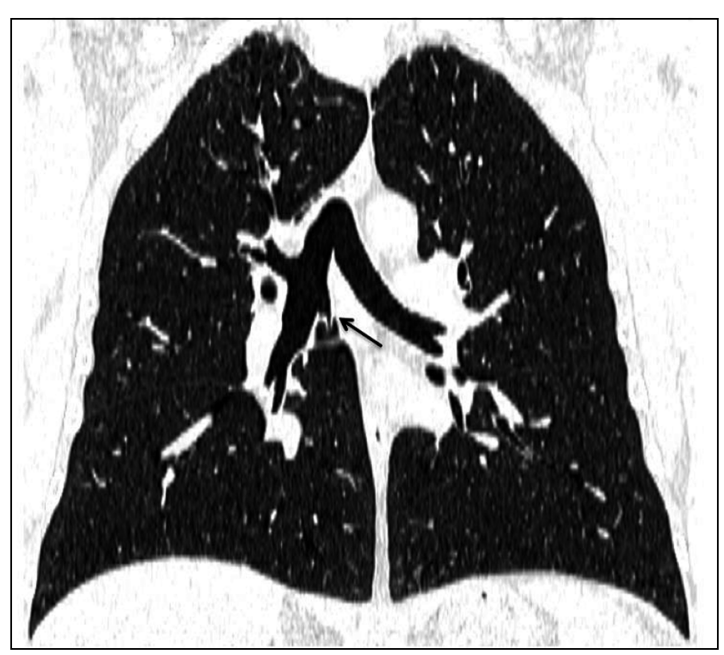

Figura 2. Reconstrucción coronal en ventana pulmonar. Pequeña estructura bronquial emergiendo desde el bronquio intermedio (flecha). 

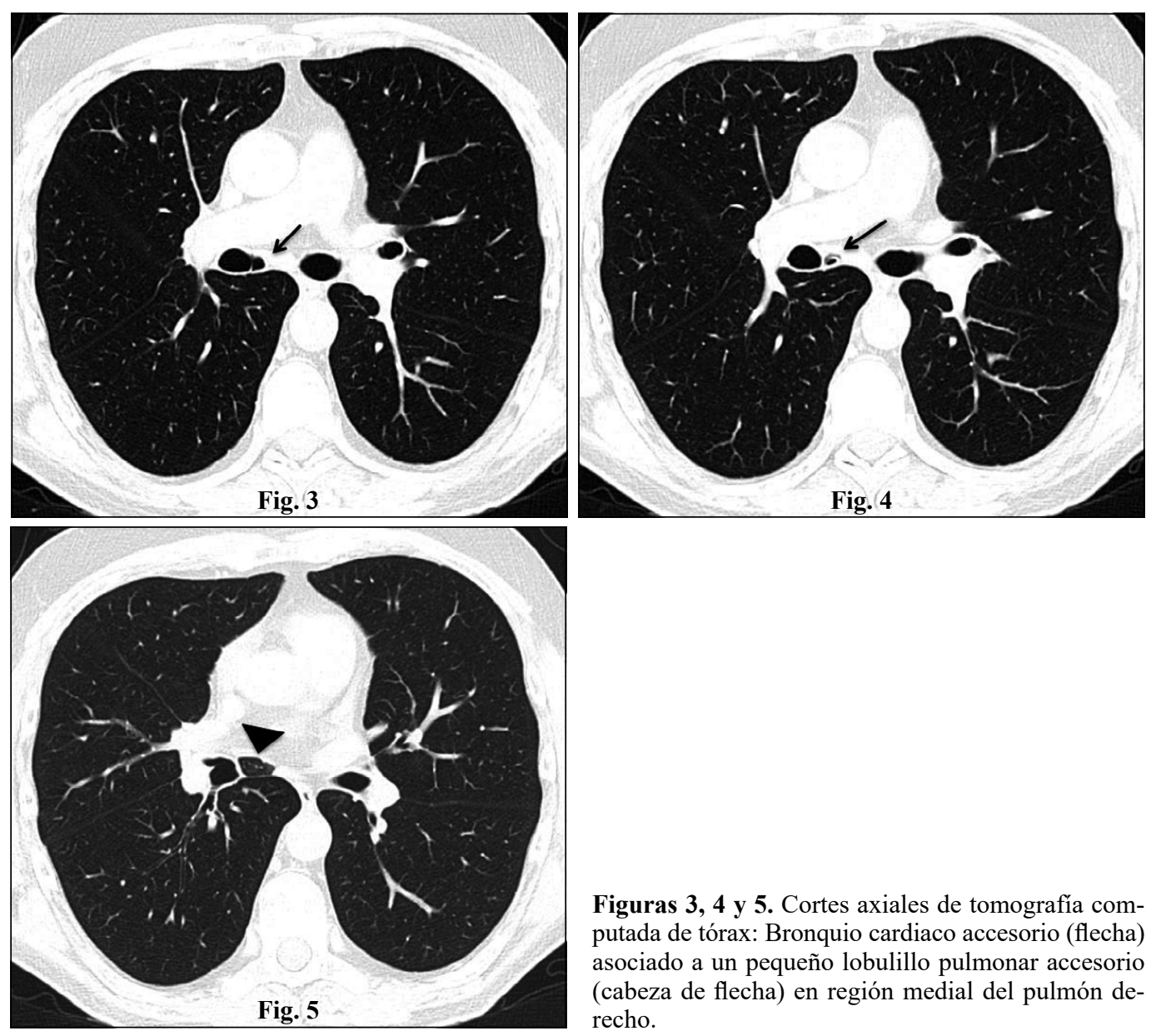

Figuras 3, 4 y 5. Cortes axiales de tomografía computada de tórax: Bronquio cardiaco accesorio (flecha) asociado a un pequeño lobulillo pulmonar accesorio (cabeza de flecha) en región medial del pulmón derecho.
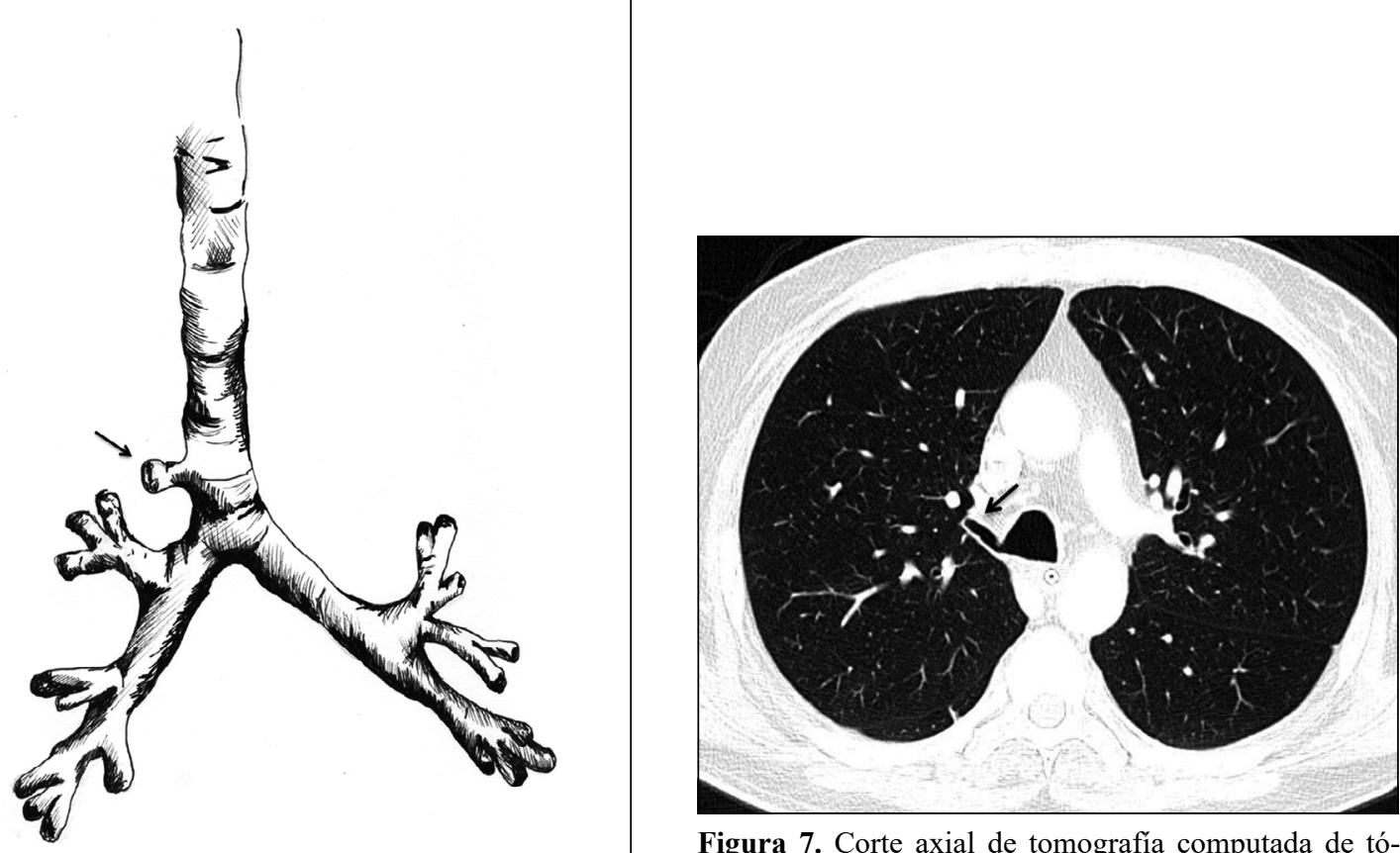

Figura 7. Corte axial de tomografía computada de tórax. Bronquio traqueal para el lóbulo superior derecho (flecha).

Figura 6. Esquema: Bronquio traqueal (flecha). 


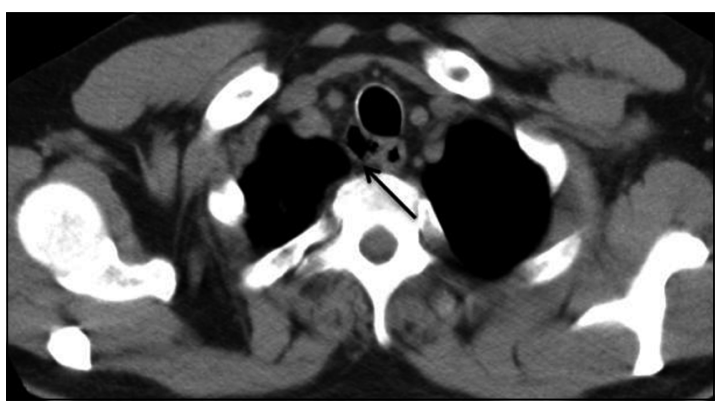

Figura 8A. Corte axial de tomografía computada de tórax en ventana mediastínica. Divertículo traqueal (flecha).

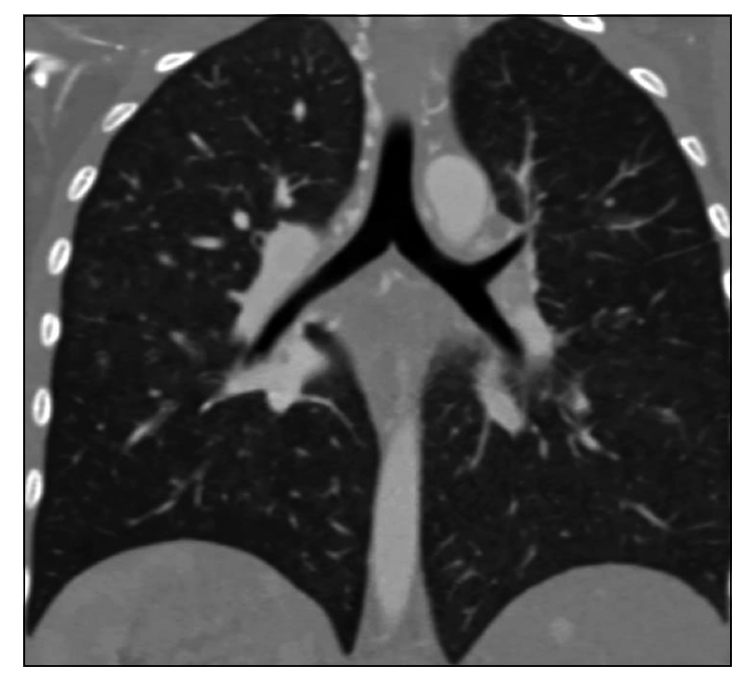

Figura 9. Reconstrucción coronal de tomografía computada de tórax. Situs inversus con inversión de la vía aérea.

El tratamiento de los bronquios ectópicos o supernumerarios habitualmente es conservador, pero en pacientes con infecciones recurrentes o hemoptisis puede ser necesaria su resección quirúrgica ${ }^{5,8,9,15}$.

\section{Isomerismo bronquial}

El isomerismo bronquial se refiere a la presentación de una anatomía bronquial simétrica, de lado derecho (asociada con asplenia) o de lado izquierdo (asociada con polisplenia), ambas relacionadas con cardiopatías congénitas y otras anomalías ${ }^{16}$.

\section{Situs inversus}

También se puede producir una inversión de la vía aérea como en el caso del situs inversus ${ }^{17}$ (Figuras 9 y 10).

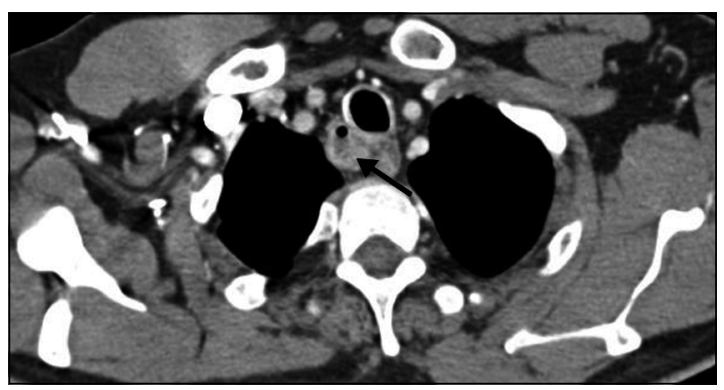

Figura 8B. Se realiza nueva TAC a los 2 meses por cuadro febril y síntomas irritativos de vía aérea superior donde se observa engrosamiento de la pared del divertículo, contenido hidroaéreo en su lumen y cambios inflamatorios del tejido adiposo adyacente (flecha).

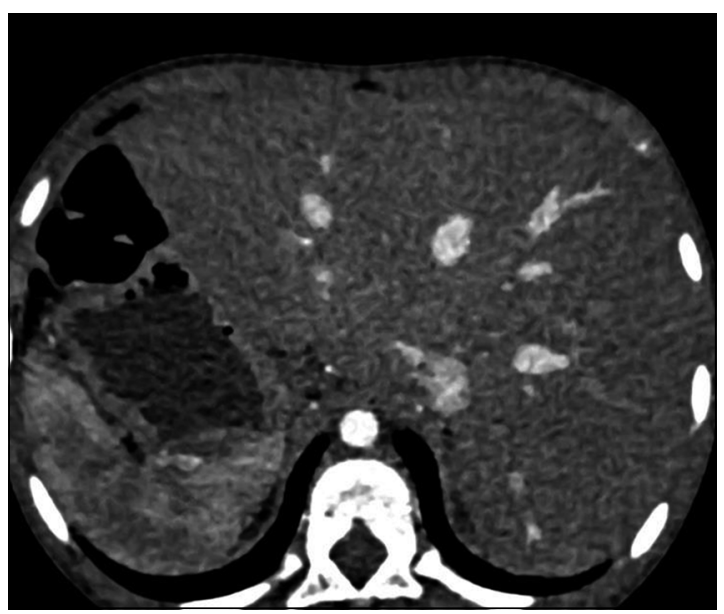

Figura 10. Corte axial de tomografía computada de abdomen superior del mismo paciente de la figura 9, con inversión de la anatomía abdominal.

\section{Bronquio puente}

Otra variante de la anatomía extremadamente rara es el bronquio puente, en que el bronquio del lóbulo inferior derecho nace del bronquio principal izquierdo y cruza el mediastino hacia el pulmón derecho ${ }^{18}$ (Figura 11). También se ha descrito una variante en que el pulmón derecho está completamente ventilado por este bronquio displásico. En ambos casos, la carina se encuentra en un nivel normal, frente a las vértebras T4 y $\mathrm{T} 5$, presentando una pseudocarina a nivel T6$\mathrm{T} 7^{19}$.

El bronquio puente se puede asociar, entre otras alteraciones, a un cabestrillo de la arteria pulmonar izquierda (también conocido como sling de la arteria pulmonar), una anomalía vascular rara en que la arteria pulmonar izquierda se origina de la arteria pulmonar derecha, pasa por encima del bronquio derecho y entre la tráquea y 


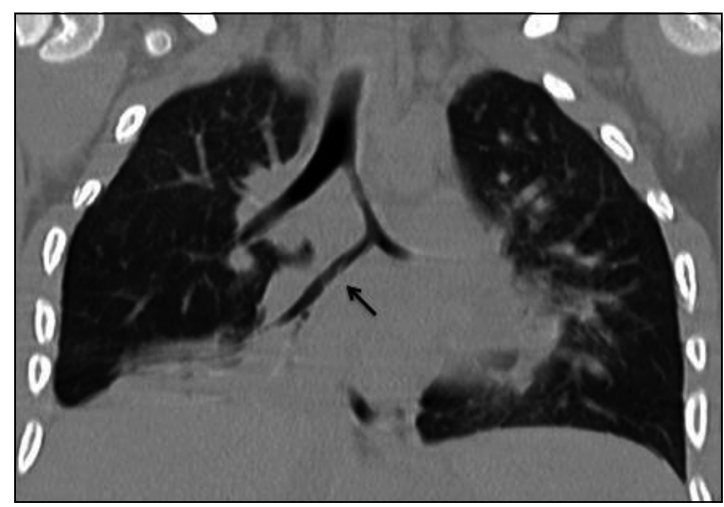

Figura 11. Reconstrucción coronal de tomografía computada de tórax. Bronquio puente que cruza el mediastino hacia el pulmón derecho (flecha).

el esófago, para ingresar al hemitórax izquierdo, lo que se puede acompañar de compresión de la vía aérea, estenosis traqueal y traqueomalacia ${ }^{19-21}$.

\section{Conclusiones}

A pesar de que estas variaciones congénitas del árbol tráqueobronquial se presentan con baja frecuencia, hay varias implicancias clínicas relacionadas con el conocimiento completo de la anatomía tráqueo-bronquial y su correcta identificación. Especial consideración se debe tener al realizar intubaciones endotraqueales, fibrobroncoscopías, lavado broncoalveolar, biopsias, tratamientos endobronquiales, instalación de stent, braquiterapia y cirugías pulmonares, entre otras intervenciones y procedimientos. Aunque existen varias modalidades de estudio disponibles, la tomografía computada es el examen de elección para el estudio anatómico de la vía aérea.

\section{Agradecimientos}

Especial agradecimiento a María Pía Carrasco por sus ilustraciones (Figuras 1 y 6 ).

\section{Bibliografía}

1.- GHAYE B, SZAPIRO D, FANCHAMPS J M, DONDELINGER R F. Congenital bronchial abnormalities revisited. Radiographics 2001; 21: 105-19.

2.- EVANS J A. Aberrant bronchi and cardiovascular anomalies. Am J Med Genet 1990; 35: 46-54.

3.- RUCHONNET-METRAILLER I, ABOU TAAM R,
DE BLIC J. Presence of tracheal bronchus in children undergoing flexible bronchoscopy. Respir Med England 2015; 109: 846-50.

4.- MANGIULEA V G, STINGHE R V. The accessory cardiac bronchus. Bronchologic aspect and review of the literature. Dis Chest 1968; 54: 433-6.

5.- MCGUINNESS G, NAIDICH D P, GARAY S M, DAVIS A L, BOYD A D, MIZRACHI H H. Accessory cardiac bronchus: $\mathrm{CT}$ features and clinical significance. Radiology 1993; 189: 563-6.

6.- JACKSON G D, LITTLETON J T. Simultaneous occurrence of anomalous cardiac and tracheal bronchi: a case study. J Thorac Imaging 1988; 3: 59-60.

7.- LAZCANO HERNÁNDEZ E, GUERRERO MARILES E D S, VELÁZQUEZ SERRATOS J R, JUÁREZ HERNÁNDEZ F, SILVA ALVARADO M, FLORES HERNÁNDEZ S S et al. Variantes bronquiales anatómicas raras. Comunicación de tres casos. Rev Inst Nac Enfermedades Respir 2007; 20: 119-25. Disponible en: http://www.medigraphic.com/pdfs/iner/in-2007/in072e. pdf (consultada en abril de 2017).

8.- ENDO S, SAITOH N, MURAYAMA F, SOHARA Y, FUSE K. Symptomatic accessory cardiac bronchus. Ann Thorac Surg 2000; 69: 262-4.

9.- BERROCAL T, MADRID C, NOVO S, GUTIÉRREZ J, ARJONILLA A, GÓMEZ-LEÓN N. Congenital anomalies of the tracheobronchial tree, lung, and mediastinum: embryology, radiology, and pathology. Radiographics 2004; 24: e17.

10.- O'SULLIVAN B P, FRASSICA J J, RAYDER S M. Tracheal bronchus: a cause of prolonged atelectasis in intubated children. Chest 1998; 113: 537-40.

11.- KUO C W, LEE Y C, PERNG R P. Tracheal bronchus associated with lung cancer: a case report. Chest 1999; 116: 1125-7.

12.- LEE E Y, GREENBERG S B, BOISELLE P M. Multidetector computed tomography of pediatric large airway diseases: state-of-the-art. Radiol Clin North Am 2011; 49: 869-93.

13.- RITSEMA G H. Ectopic right bronchus: indication for bronchography. Am J Roentgenol 1983; 140: 671-4.

14.- DOOLITTLE A M, MAIR E A. Tracheal bronchus: classification, endoscopic analysis, and airway management. Otolaryngol Head Neck Surg 2002; 126: 240-3.

15.- FREEMAN S J, HARVEY J E, GODDARD P R. Demonstration of supernumerary tracheal bronchus by computed tomographic scanning and magnetic resonance imaging. Thorax 1995; 50: 426-7.

16.- APPLEGATE K E, GOSKE M J, PIERCE G, MURPHY D. Situs revisited: imaging of the heterotaxy syndrome. Radiographics 1999; 19: 834-7.

17.- LANDING B H, LAWRENCE T Y, PAYNE V C J, WELLS T R. Bronchial anatomy in syndromes with abnormal visceral situs, abnormal spleen and congenital heart disease. Am J Cardiol 1971; 28: 456-62.

18.- STARSHAK R J, STY J R, WOODS G, KREITZER F 
V. Bridging bronchus: a rare airway anomaly. Radiology 1981; 140: 95-6.

19.- WELLS T R, GWINN J L, LANDING B H, STANLEY $\mathrm{P}$. Reconsideration of the anatomy of sling left pulmonary artery: the association of one form with bridging bronchus and imperforate anus. Anatomic and diagnostic aspects. J Pediatr Surg 1988; 23: 892-8.

20.- ZHONG Y M, JAFFE R B, ZHU M, GAO W, SUN
A M, WANG Q. CT assessment of tracheobronchial anomaly in left pulmonary artery sling. Pediatr Radiol 2010; 40: 1755-62.

21.- LEE K H, YOON C S, CHOE K O, KIM M J, LEE $\mathrm{H}$ M, YOON H K et al. Use of imaging for assessing anatomical relationships of tracheobronchial anomalies associated with left pulmonary artery sling. Pediatr Radiol 2001; 31: 269-78.

Correspondencia a:

Dra. Tamara Palavecino B.

Hospital Clínico Universidad de Chile

Santiago, Chile

Santos Dumont 999, Santiago.

Email: tamaritz@gmail.com 